\title{
Portal hypertension caused by partial nodular transformation of the liver
}

\author{
M. ClASSEN, K. ElSTER, H. J. PESCH, AND L. DEMLING \\ From the Departments of Medicine and Pathology, University of Erlangen and Nuremberg, Germany
}

SUMmARY The course of the disease in, and the necropsy on, a 46-year-old woman with partial nodular transformation of the liver are described. This is, as far as is known, the fifth case to be reported. On the basis of results obtained from clinical tests partial nodular transformation of the liver was assumed. Seven months after the first episode of haematemesis and after numerous haemorrhages, the patient died. The morphological picture of the liver confirmed the tentative diagnosis. In spite of opinions to the contrary (Ingelfinger, 1967), the clinical picture of portal hypertension, can, by reason of the morphological findings, be clearly differentiated from hepatic cirrhosis and other nodular changes in the liver.

\section{Case Report}

A 46-year-old housewife had had epigastric complaints for several years and, during the few months before admission to hospital, additionally noticed some loss of weight and appetite. There was no history of hepatitis, nor was there any question of alcohol abuse. In February 1968 the patient had a sudden haematemesis and was admitted to hospital. Radiological evidence of oesophageal varices was found. A liver specimen, obtained by percutaneous needle biopsy, showed slight damage to the parenchyma, accompanied by sparse necrotic areas, moderate activity of the parenchyma, periportal fibrosis, and central lobular sclerosis. In May 1968 the patient was admitted to this hospital for further diagnosis. The patient had a slightly jaundiced skin and sclerae and palmar erythema, but no spider naevi. The liver edge was $3 \mathrm{~cm}$ below the right-hand portal margin. The spleen was considerably enlarged and ascites was present.

\section{LABORATORY FINDINGS}

Our analyses showed the following: $\mathrm{Hb} 10.3 \mathrm{~g} \%$, 2 million erythrocytes, $\mathrm{Hb}_{\mathrm{E}} 36$, haematocrit $32 \%$, 1,700 leucocytes, 140,000 platelets, $26 \%$ reticulocytes; bilirubin, $3.5 \mathrm{mg} \%$; alkaline phosphatase, $270 \mathrm{mU}$, GOT $36 \mathrm{mU}$, GPT $11 \mathrm{mU}$,
LDH $110 \mathrm{E}$, Fe 89\%; cholesterol $170 \mathrm{mg} \%$, urea $\mathrm{N} 31 \mathrm{mg} \%$, total serum protein $7.8 \mathrm{mg} \%$, and a normal electrophoretic pattern.

Examination of the sternal marrow indicated a splenogenous medullary inhibition of myelopoiesis.

\section{RADIOLOGICAL EXAMINATIONS}

The state of the oesophageal varices is shown in Figure 1. There was a prominent axial sliding hiatal hernia, and the stomach was displaced by an enlarged spleen, and a pedicled duodenal polyp in the descending arm.

The result of splenoportography is shown in Figure 2. There was a selective puncture of the spleen during peritoneoscopy; no obstruction in the portal vein was visible. An abrupt reduction in the contrast density of the small hepatic branches could be seen. The gastrooesophageal veins had a convolution on a level with the fundus of the stomach, showing the presence of varices.

The pressure in the portal vein was $23 \mathrm{~mm} \mathrm{Hg}$, and in the wedged hepatic vein there was a pressure of $13 \mathrm{~mm} \mathrm{Hg}$.

ENDOSCOPIC AND HISTOLOGICAL

Gastroscopy presented no evidence of an ulcer 

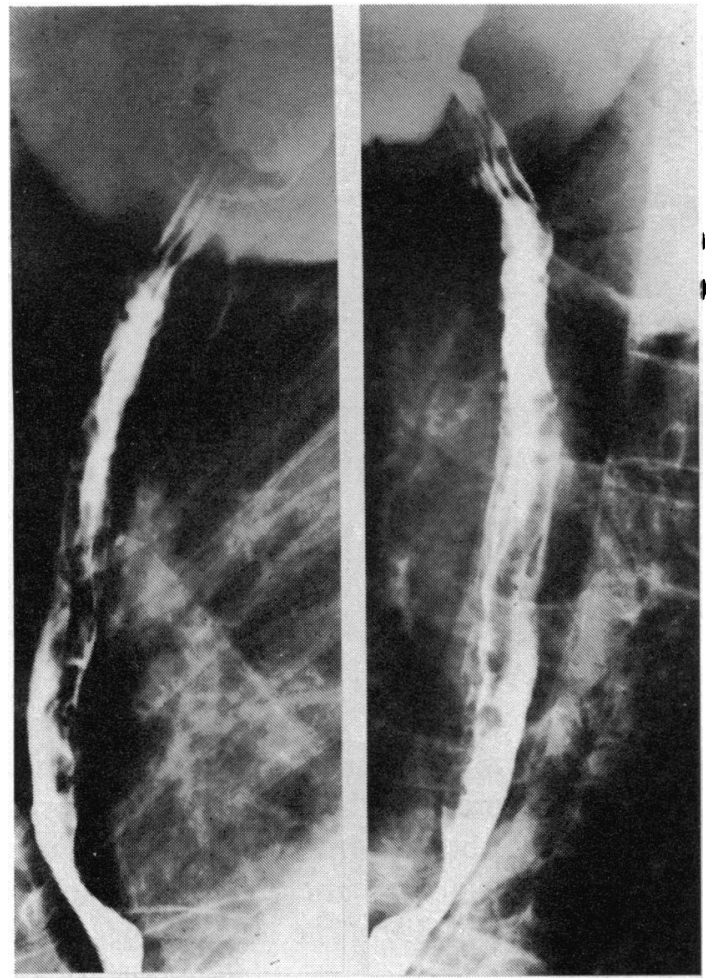

Fig. 1 Oesophageal varices.

or other source of haemorrhage in the stomach. Oesophagoscopy showed considerable varices in the lower third, and peritoneoscopy indicated an enlargement of both lobes of the liver, which showed a smooth surface and normal colouring. There was considerable enlargement and an increase in the density of the spleen. Tortuous and enlarged mesenteric vessels were apparent. Two biopsy punctures of the left lobe of the liver were carried out.

\section{Histological examination of the liver tissue}

A localized increase in the number of Kupffer cells was shown. The periportal tracts varied: some were narrow and poor in cells, and others clearly extended and rich in cells. No cirrhotic changes were observed. At this stage we diagnosed a localized activation of the mesenchyma.

\section{Ultrasonic scanning image}

The reflexion pattern (Fig. 3) obtained here is similar to that produced by cirrhotic liver, where the structure also produces a consistently non-homogeneous pattern. However, the picture, when compared with that presented by a cirrhotic liver, is not complete, for the liver surface is smooth and free from the characteristic nodularity, and the coarsening or thickening of the lower edge of the liver is absent (Dr Rettenmaier).

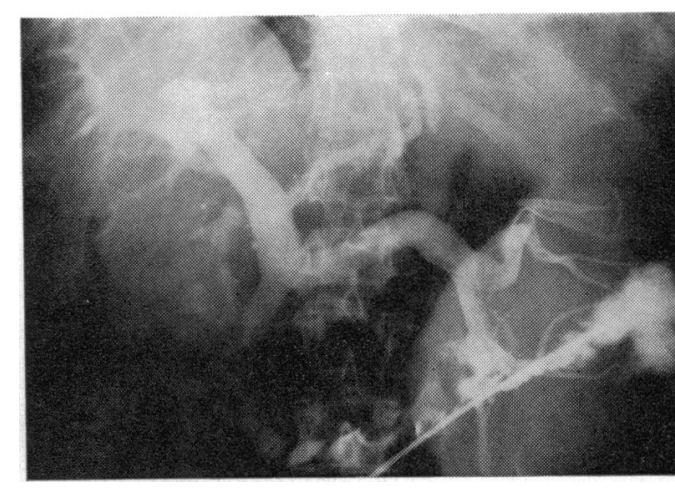

Fig. 2 Splenoportography: gastrooesophageal collateral circulation.

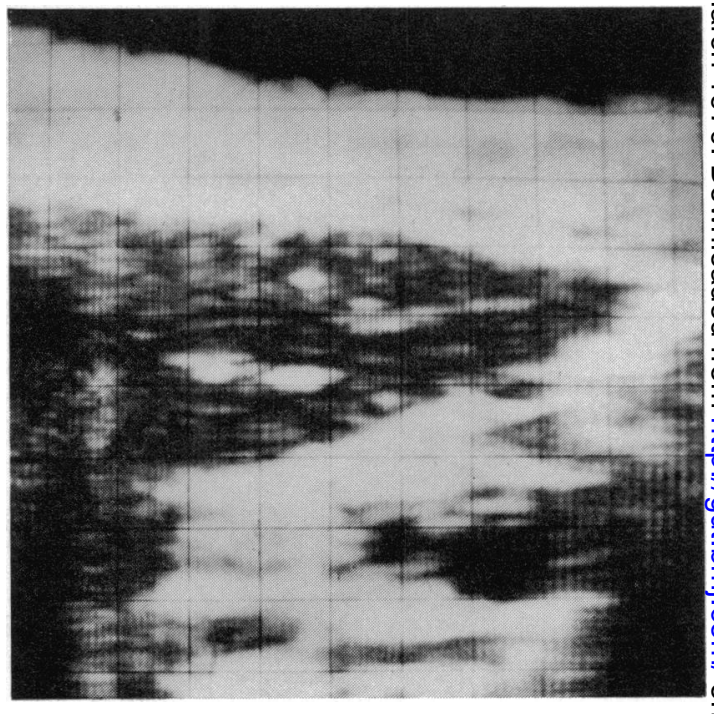

Fig. 3 Ultrasonic scan: longitudinal section in the midline. Left hand edge of image = cranial, right hand of image = ventral. One side of the graticule corresponds to $1 \mathrm{~cm}$ (Dr Rettenmaier).

Scintigraphy of the liver using $100 \mu \mathrm{Ci}{ }^{198} \mathrm{Au}$ 罗 colloidal gold

This indicates a moderately enlarged liver with an uneven distribution of activity, and an in- $\stackrel{?}{?}$ crease in the enlarged spleen.

During the patient's month in hospital before the operation, three massive haemor- $\stackrel{\mathbb{Q}}{\stackrel{Q}{Q}}$ rhages from varices occurred, with blood losses $\stackrel{\otimes}{\varnothing}$ ranging from 500 to $1,500 \mathrm{ml}$. Bleeding was con- $\bar{\sigma}$ trolled by transfusions, the administration of vasopressin and haemostyptics, and the $\frac{0}{0}$ use of the Sengstaken tube. The ascites was washed out with Furosemid and Triamteren, $\frac{\bar{c}}{\overrightarrow{2}}$ the serum electrolytes remaining stable. After the examinations had been concluded, a laparotomy was performed. Because of massive oeso- 
phageal variceal haemorrhage occurring during the operation, all that could be done was to insert a Vossschulte ring and to remove a lymphatic node from the porta hepatis. No wedge biopsy specimen was removed from the liver on this occasion. For the first six weeks after the operation the patient showed signs of making a good recovery and no bleeding was observed. The liver function remained stable and the ascites, in response to treatment with drugs, largely disappeared. After four massive haemorrhages in August, the patient finally died in September 1968 as a result of cardiac failure.

\section{Anatomical-pathological Findings in the Liver}

\section{MACROSCOPIC FINDINGS}

The weight of liver was $1,700 \mathrm{~g}$ and there were clear indications of increased density and of somewhat non-homogeneous consistency. The colour was reddish-brown, the surface smooth and showing several band-like capsular adhesions on the ventral surface. On the cut surface (Fig. 4), which was of the same colour, a number of dilated vessels in the centre of numerous polygonal nodules were observed. The adjacent parenchyma protruded above the periphery of the nodules. The diameter of the nodules was 1.2 to $3 \mathrm{~cm}$. Around several of the central vessels, the connective tissue was obviously increased. A considerable increase in connective tissue was observed in the region about the hilus of the liver. On cutting into the tissue with a knife, green thread material was seen (the condition as after the operation). The vena cava and the portal veins were normally situated. No appreciable constriction of the lumina was to be seen. The intima of the portal vein, as well as of the afferent and

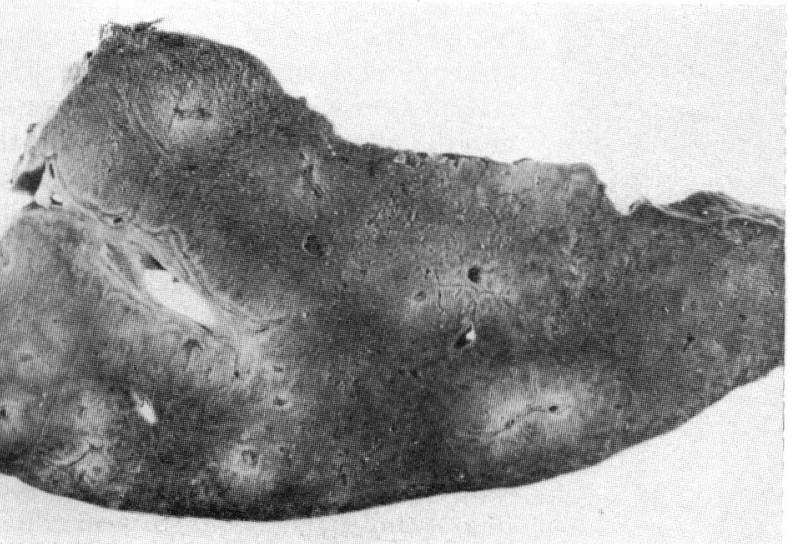

Fig. 4 Cross section of the liver, in which many nodules can be seen. efferent branches, was markedly sclerotic, and diffuse and confluent, grey-white plaques could be seen.

\section{MICROSCOPIC FINDINGS}

In sections taken from the hilum of the liver, widespread fibrotic, interlaced 'calloused' strands were observed and loose, thread-like infiltrations comprising lymphocytes and plasma cells were discovered between these strands. In the 'calloused' tissue a medium sized vessel had a 'filling' of tissue with considerable granular deposition of haemosiderin in its lumen. The parietal layers were so disintegrated that no reliable statement could be made as to the nature of the vessel. The calloused strands radiated into the liver parenchyma so that the resulting histological picture of intralobular fibrosis showed an atrophy of the trabeculae. Such changes were, however, recognizable only in the immediate vicinity of the hilum (as a consequence of the operation). The nodular parenchyma showed a reversal of structure as compared with normal liver tissue, which was composed of epithelial trabeculae arranged approximately radially, in the centre of which Glisson's triads (some obviously extended) with dilated branches of the portal vein were detected (Figure 5). Moreover, within the nodules, the number of branches of the hepatic vein were considerably reduced. The parenchyma adjacent to the nodules manifested varying degrees of atrophy (Figure 6). Further away from the nodules, the predominant picture was one of subchronic vascular congestion. In these areas the periportal spaces were, in part, extended and showed an increase in fibrous tissue. In the surrounding areas, the periportal spaces contained several, noticeably wider bile ducts, and ductular proliferation. The intrahepatic branches of the portal vein showed either a wide lumen or thickened walls. In the neighbouring parenchyma, narrow epithelial trabeculae with wide sinusoids full of erythrocytes were seen. In a number of sections the basic reticular network showed superficial changes into delicate collagenous fibres. A change in thestructure itself, however, had not taken place.

The final anatomical-pathological diagnosis was that a partial nodular transformation of the liver had been accompanied by atrophy of the adjacent parenchyma. In the centre of the nodules there was extreme periportal fibrosis, which was also seen in various parts of the peripheral parenchyma (Figures 5 and 6). In addition, there was scarred and calloused hilar fibrosis. The portal vein was sclerotic. The liver weighed $1,700 \mathrm{~g}$ and there was evidence of subchronic vascular congestion. There was a chronic congestion of the spleen (weight $1,400 \mathrm{~g}$ ) with capsular fibrosis and localized, myeloid metaplasia, and evidence of older and new haemorrhages from oesophageal varices. 


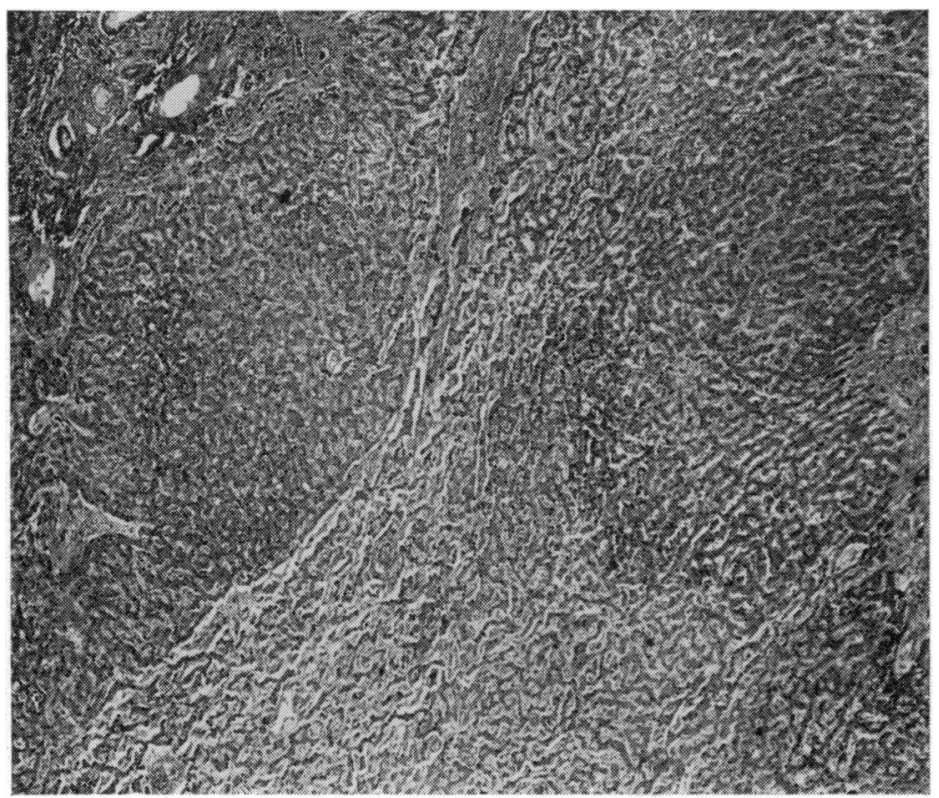

Fig. 5 Parts of two dilated portal triads with broad biliary ducts and vessels. Surrounding tissue 'nodular transformed'. Stained $H E \times 45$.

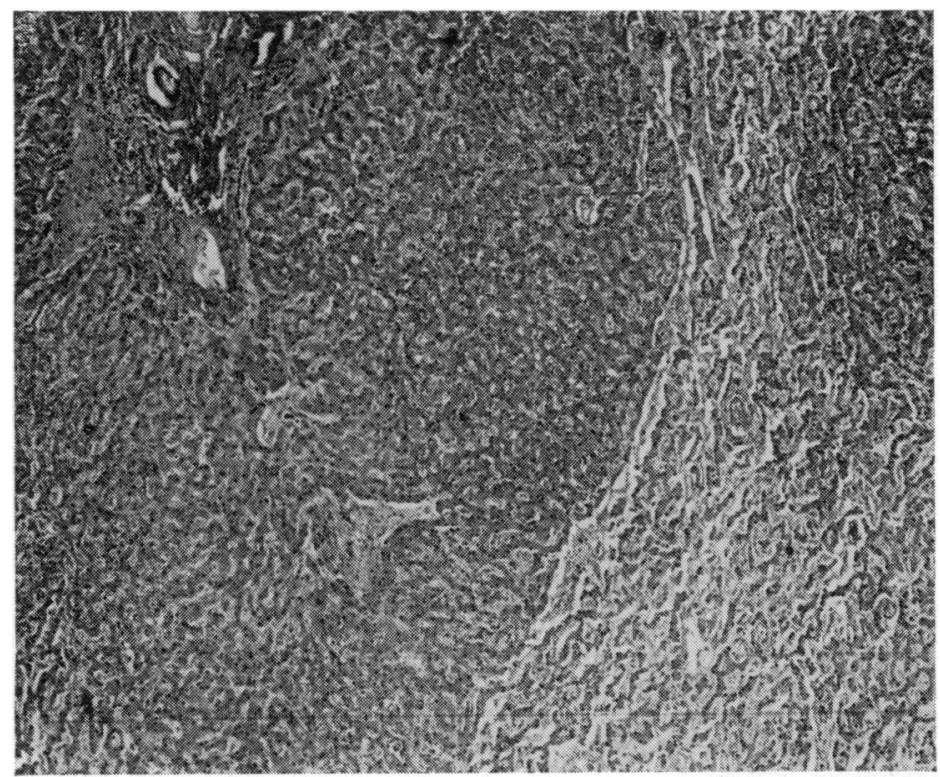

Fig. 6 Atrophy of parenchyma around a nodule. Stained $H E \times 60$.

\section{Discussion}

DIFFERENTIAL DIAGNOSIS

Haematemesis or the discovery of splenomegaly is the first serious symptom of this disease. Only in one previous case, as described by Sherlock, and now in this patient, was there previous, unexplained epigastric pain. Here the former course of the disease was also characterized by episodes of bleeding, which finally led to the death of the patient. Between the first episode of bleeding from oesophageal varices and death, as many as 16 years can pass (Sherlock, 1968) if a portocaval anastomosis is carried out.

The liver function tests are as unspecific as the somatic findings; the clinical findings and the results of the laboratory tests thus provided as few differential diagnostic pointers as the liver needle biopsy specimens.

Splenoportography indicated the presence of portal hypertension with a portogastrooesophageal shunt and a considerably reduced contrast of the intrahepatic branches of the portal vein, changes which pointed to a cirrhosis of the liver.

The procedure of peritoneoscopy makes possible not only the carrying out of selective needle biopsies, but also the simultaneous measurement of intrasplenic pressure and the radiological representation of the portal venous system. We consider it to be highly important in those cases where organs are subject to high portal venous pressure that the site of the needle biopsy can be visually controlled and secondary bleeding treated electrothermically. In experienced hands the possibility of being able to combine these three important examinations in one procedure would reduce both the complexity of examination method, and risk to the patient. Moreover, peritoneoscopy was significant in our differential diagnosis. Apart from the considerable congestion of the mesenteric vessels and splenomegaly, there was merely slight hepatomegaly not accompanied by any visible signs of a cirrhosis. The histological picture presented by needle biopsy specimens of the liver did not indicate the presence of a cirrhotic condition. This result, in conjunction with the results of liver scintigraphy and the ultrasonic scan, allowed for the first time the possibility of a noncirrhotic, nodular transformation of the liver. This being the suspected diagnosis the patient was then referred to the surgeons for operation. The desired removal of a wedge biopsy specimen from the liver, which can be decisive for the diagnosis, could not be effected so that our tentative diagnosis was not verified until a postmortem examination had been carried out. In life, a specimen obtained by wedge biopsy, rather than needle biopsy, seems capable of assisting diagnosis. If surgical relief of the pressure in the high portal circulation can be provided early enough, the prognosis would seem better than in a case of hepatic cirrhosis (Sherlock, 1968). 
HISTOLOGICAL FINDINGS

The histological changes found in the liver of our patient corresponded exactly with those described by Sherlock et al (1966) and Sherlock (1968). The nodules, which were recognizable macroscopically, showed reverse lobulation with extended periportal spaces, dilated portal vein branches and a reduced number of branches of the hepatic vein. The parenchyma surrounding the nodules was atrophic; elsewhere, fibrosis, proliferation of the ductules, and inflammation were merely locally represented. Any compression or thrombosis of the portal vein and its two large branches, where there was considerable fibrosis and sclerosis of the walls, was absent. The increase in connective tissue in the vicinity of the porta hepatis had no connexion with the nodular transformation. In our opinion, this phenomenon must be seen in the light of the surgical intervention performed in this area at an earlier date.

\section{The February 1970 Issue}

\section{THE FEBRUARY 1970 ISSUE CONTAINS THE FOLLOWING PAPERS}

Propulsion (mass movements) in the human colon and its relationship to meals and somatic activity D. J. HOLDSTOCK, J. J. MISIEWICZ, T. SMITH, and E. N. ROWLANDS

Factors controlling colonic motility: Colonic pressures and transit after meals in patients with total gastrectomy, pernicious anaemia, or duodenal ulcer D. J. HOLDSTOCK and J. J. MISIEWICZ

A study of abnormal muscular patterns in diverticular disease of the colon using the polysiloxane foam enema L. E. HUGHES

The neurological junction between the appendix and ascending colon JOHN L. EMERY and JANET UNDERWOOD

Rectal and colonic studies after resection of the sigmoid for diverticular disease T. G. PARKS

Bile salt secretion in cirrhosis of the liver LESLIE A. TURNBERG and GORDON GRAHAME

Effects of rose bengal on bile secretion in the rabbit: inhibition of a bile salt-independent fraction D. DHUMEAUX, S. ERLINGER, J.-P. BENHAMOU, and R. FAUVERT

Cholelithiasis in Singapore: Part I A necropsy study W. S. HWANG

Part II A clinical study H. K. CHIAM, P. N. UNNI, and W. S. HWANG

Maximal acid response of Pavlov pouches to food and histamine A. MARVIN BROOKS and MORTON I. GROSSMAN

\section{CIRCULATORY STUDIES}

The obvious rise in presinusoidal pressure, in association with the moderately increased wedged hepatic vein pressure, does not admit of any clear classification. On the basis of earlier examinations (Sherlock et al, 1966), it was considered that portal hypertension, caused by non-cirrhotic transformation of the liver, was of the postsinusoidal type, being in turn caused by the reduction in the hepatic vein and the compression of the radicles by the nodules which thus disturbed the intrahepatic circulation.

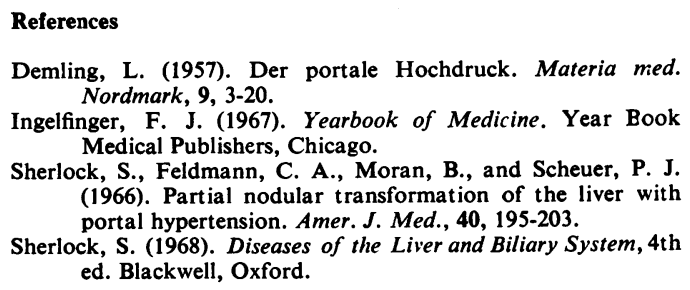
Nordmark, 9, 3-20.

Ingelfinger, F. J. (1967). Yearbook of Medicine. Year Book Medical Publishers, Chicago.

Sherlock, S., Feldmann, C. A., Moran, B., and Scheuer, P. J. (1966). Partial nodular transformation of the liver with portal hypertension. Amer. J. Med., 40, 195-203.

Sherlock, S. (1968). Diseases of the Liver and Biliary System, 4th ed. Blackwell, Oxford. 